\title{
Characteristic Analysis of Transient Electromagnetic Disturbance Data in Substation
}

\author{
Xin $\mathrm{Wu}^{1}$, Shu $\mathrm{Li}^{2}$ \\ ${ }^{1}$ School of Electrical and Electronic Engineering, North China Electric Power University, Beijing, China \\ ${ }^{2}$ Institute of Medical Devices Control, National Institutes for Food and Drug Control, Beijing, China \\ Email: 19861002wuxin@163.com
}

Received March 2015

\begin{abstract}
Characteristic analysis of transient electromagnetic disturbance data is very important. In this paper, the time-domain and frequency-domain characteristics of transient electromagnetic disturbance caused by switching operations in substations are analyzed. Two methods are adopted in time-domain analysis. One method is histogram and the other one is normality test. An appropriate procedure for the normality test is proposed. Furthermore, the method used in frequencydomain analysis is to obtain overall envelope. These statistical characteristic analysis methods are showed to be of great value in analyses of the measured samples. The simulation results showed that these methods are effective.
\end{abstract}

\section{Keywords}

Transient Electromagnetic Disturbance, Time-Domain Characteristic, Frequency Domain Characteristic, Normality Test

\section{Introduction}

With the increasing requirements of the electromagnetic environment, electromagnetic compatibility problems have become the focus of research in electrical and electronic field. In power system, the complex and advanced equipment cause electromagnetic disturbance produced a more complex, but the ability of advanced equipment tolerance of electromagnetic disturbance weaker, so the electromagnetic compatibility is an important factor affecting power system safe and reliable production [1]. Electromagnetic compatibility problems appear most substation which is the main object of study, transient electromagnetic disturbance caused by lightning strike, fault and switching operation easily through the coupled radiation conduction on the formation of interference two equipment, cause two equipment damage [2] [3].

With the time domain and frequency domain characteristics of complex electromagnetic transient analysis of time domain characteristics of harassment, is helpful to obtain harassment amplitude changes with the time and frequency domain analysis of characteristics in order to provide evidence for shielding and filtering of the two 
device, ensure the normal work of its own [4]-[6]. In this paper, transient electromagnetic disturbance produced by switching operations in substation to analyze and study, according to the features of time domain and frequency domain for transient electromagnetic disturbance of the actual measurement data was analyzed, the overall characteristics of transient electromagnetic disturbance is obtained. Because the data is difficult to obtain, the number of samples is small, this paper focuses on the small sample statistical theory was proposed based on the normality test method of time domain characteristics. In addition, based on the practical consideration, according to the statistical results of the evaluation of the method used by the meaning and practical value.

\section{Analysis of Characteristic Parameter}

All kinds of transient electromagnetic disturbance quantity substation measure and can be used to evaluate various characteristic parameters, including time domain and frequency domain parameters [7]. Below the basic parameters used in this paper are briefly explained as follows.

1) Micro pulse rise (fall) time $T_{r}$ : Distance between the instantaneous peak corresponding to the time of the recent peak of $10 \%$ and then reached the $90 \%$ peak instantaneous time interval, is the peak corresponding to the rise time, fall time of negative peak corresponding to the fall time

2) Micro pulse duration $T_{m}$ : The transient wave: start time and end time.

3) The peak to peak value of $U_{m}$ : The absolute duration positive peak and a negative peak difference.

4) The frequency band width of $B_{W}$ : This article from the perspective of immunity test to define the electric field strength is higher than that of $1 \mathrm{~V} / \mathrm{m}$, magnetic field intensity is higher than that of $2.65 \mathrm{~mA} / \mathrm{m}$, voltage disturbance is higher than $1 \mathrm{~V}$, higher than the current interference frequency components included in the frequency range of $20 \mathrm{~mA}$.

5) The dominant frequency $D F$ : Interference frequency band width in the range of $B_{W}$ is higher than the reference value, amplitude frequency of $6 \mathrm{~dB}$ group.

\section{Characteristics Analysis in Time Domain}

Analysis of time domain characteristics of transient disturbance can obtain the interference source of strength and polarity characteristics and duration of important information, but also the design of an important basis for immunity test. This section of the micro pulse rise (fall) time, micro pulse duration and peak to peak were analyzed by two methods, one is the histogram statistics, the other is the normality test.

The characteristic parameters obtained by one measurement only, if you want to understand the regularity of multiple measurements to the overall analysis. This section carries on the overall statistics of the transient disturbance sample of all, and then gets the probability distribution and trend.

Figure 1 shows the probability distribution histogram about characteristic parameters of rise (fall) time and peak to peak value. Figure 1(a) is the histogram of rise (fall) time, while Figure 1(b) is peak to peak value. As can be seen from Figure 1, the characteristic parameters of probability are in a range the distribution, which can be analyzed and obtained the conclusion from it.

Transient electromagnetic disturbance data of switching operation has obvious random character. If by the time domain characteristics of the whole distribution information, the time domain characteristics can be more accurately grasp the transient disturbance data. The assumption that the distribution of the sample overall, suppose through the validation of the test method, the most common is the assumption of normality assumption. For transient electromagnetic disturbance in the substation switching operation caused by, no literature clearly points out that whether the data obey the normal distribution, so in the test of normality is very necessary.

There are several kinds of normality test methods applied to engineering. According to the design process of testing different applications need reasonable, balance each method and integration. The analysis of this paper should be used. Figure $\mathbf{2}$ is the normality flow chart of the method applied to the test.

The normal probability paper test is rough but very convenient, which can be directly on the sample to give an approximate evaluation. According to the graphic trend function transformation corresponding to the sample, it is suitable for the preliminary test. W and D tests are parallel methods, which can be used one according to the real application [8]. The two methods take parameters calculated as the judgment basis to make the conclusion. In the sample by $\mathrm{W}$ or $\mathrm{D}$ test does not meet the normal requirements of the situation. This method is suitable for the direction of test. Equation (1) and Equation (2) are the peak $\mathrm{K}$ to measure sample data deviate from a distribution. 


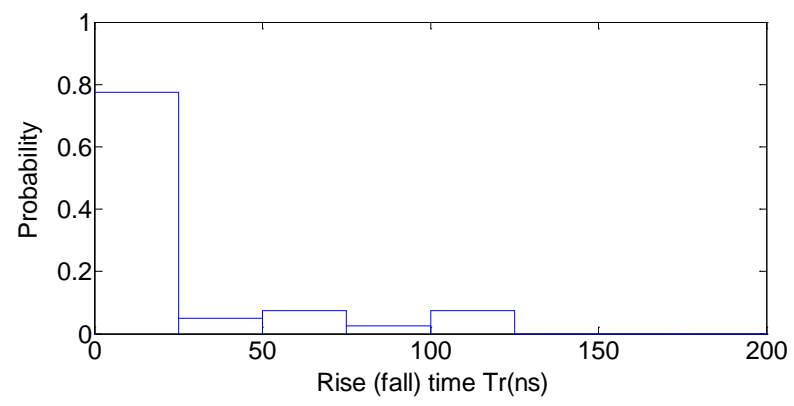

(a)

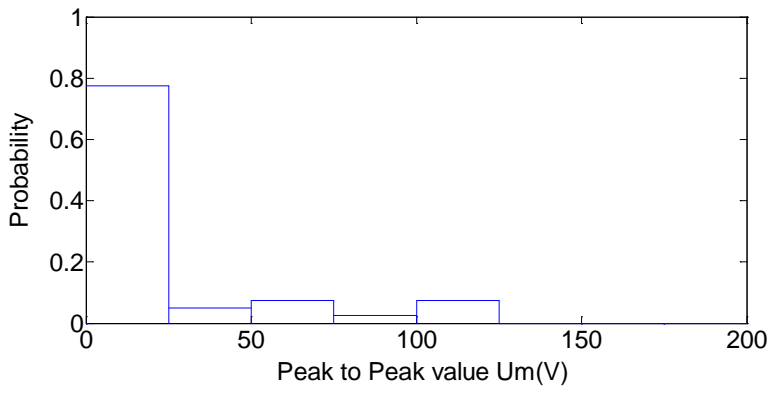

(b)

Figure 1. Probability distribution histogram characteristic parameters of time domain.

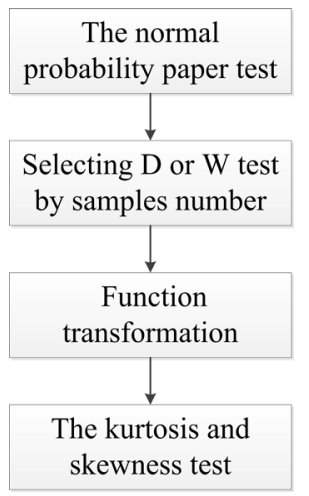

Figure 2. Normality test flow chart.

$$
\begin{aligned}
& k=\frac{E(X-\mu)^{4}}{\sigma^{4}} . \\
& v=\frac{E(X-\mu)^{3}}{\sigma^{3}} .
\end{aligned}
$$

This section of the measured data of characteristic parameters was carried out on the normality test. Figure 3 is the rise (fall) time samples were taken from the normal probability paper test chart Log function transform, known from test results, as is the logarithmic transformation can be broadly distributed in the fitting line near, can be considered approximately the normal requirements, a further test value, the other two parameters are also obtained the same results.

\section{Characteristics Analysis in Frequency Domain}

The analysis method of this section is to put all the transient disturbance data were carried on the spectrum 
analysis, and then put them together, for the spectrum amplitude of each frequency point maximum, $80 \%$ large value of envelope and 50\% large value envelope. Figure $\mathbf{4}$ is a measured sample envelope diagram in this paper. Figure 4(a) is the $80 \%$ value envelope, while Figure 4(b) is the $50 \%$ value envelope.

From Figure 4, frequency component in the 60 group of transient electromagnetic disturbance is mainly in the range of 20 - $50 \mathrm{MHz}$. In the 150 - $800 \mathrm{kHz}$ range has frequency components with larger density. By comparing the 3 kinds of envelope, know the low-frequency part component.

Density decreases obviously, in the $100 \%$ envelope energy density is the biggest, the band has been expanded to $15-50 \mathrm{MHz}$, the frequency domain analysis of transient electromagnetic disturbance is very important, in every switch operation, due to the measurement disturbance level.

\section{Acknowledgements}

This work is supported by the Fundamental Research Funds for the Central Universities of China under grant 13QN01 and the National Natural Science Foundation of China under grant 51407072. The authors wish to express their gratitude to the editor and the anonymous reviewers.

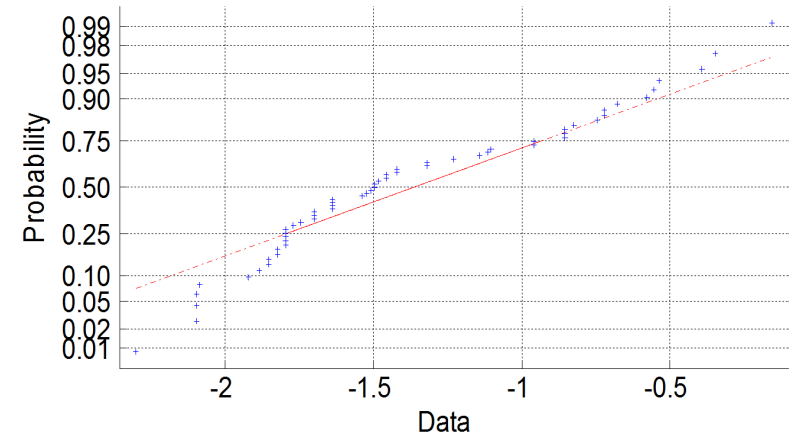

Figure 3. The normal probability paper test.

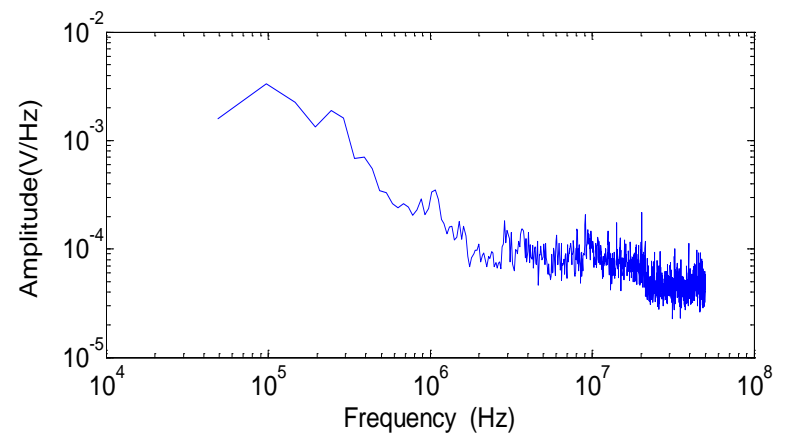

(a)

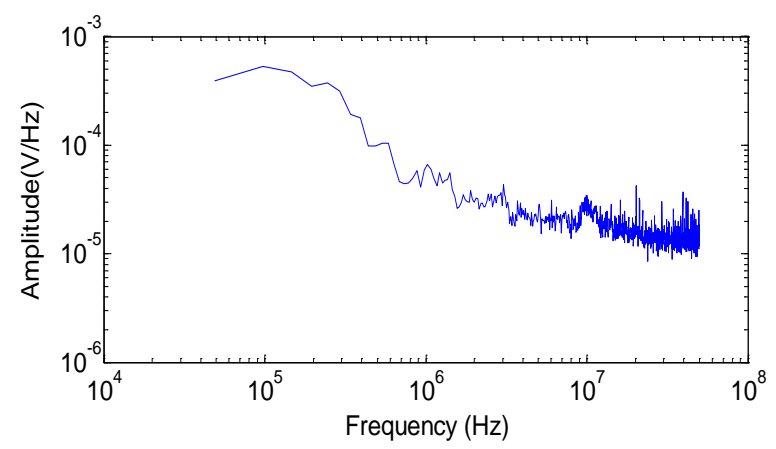

(b)

Figure 4. The envelope in frequency domain. 


\section{References}

[1] Wang, H.X. and He, J.L. (2004) Power System Electromagnetic Compatibility. Wuhan University Press, Wuhan.

[2] Sun, Z.S., Zhang, Y.F. and Zhang, G.Z. (2000) Study on EM Disturbance in 500 kV Substation and Protection Measures. High Voltage Engineering, 26, 16-18.

[3] Wiggins, C.M., Salas, T.M. and Wright, S.E. (1994) Transient Electromagnetic Interference in Substations. IEEE Transaction on Power Delivery, 9, 1869-1881. http://dx.doi.org/10.1109/61.329520

[4] Wiggins, C.M. and Wright, S.E. (1991) Switching Transient Fields in Substations. IEEE Transaction on Power Delivery, 6, 591-599. http://dx.doi.org/10.1109/61.131116

[5] Allan, G.W. and Mietek, G.W. (1998) Voltage Escalation in Vacuum Switching Operations. IEEE Transactions on Power Delivery, 3, 1698-1706.

[6] Zhang, W.D. (2003) Research on Switching Transient Electromagnetic Interference in Substations. Ph.D. Thesis, North China Electric Power University, Baoding.

[7] Du, D.Q. (2006) Compensation and Statistic Analysis of Transient Disturbance Data of Switching Operations Measured in Substations. Ph.D. Thesis, North China Electric Power University, Baoding.

[8] (1999) The Compilation of Standard Statistical Methods applied Country. Statistical Analysis and Data Processing Volume, the Statistical Interpretation of Data Normality Test. Chinese Standards Press, Beijing, 142-159. 